\title{
Five Crucial Prognostic-Related Autophagy Genes Stratified Female Breast Cancer Patients Aged 40- 60 Years
}

\section{Xiaolong Li}

Xuchang Central Hospital

Hengchao Zhang

Xuchang Central Hospital

Jingjing Liu

Xuchang Central Hospital

\section{Ping Li}

Xuchang Central Hospital

Yi Sun ( $\nabla$ sunyi19700420@163.com )

Xuchang Central Hospital

\section{Research Article}

Keywords: Autophagy genes, SERPINA1, HSPA8, HSPB8, MAP1LC3A, DIRAS3

Posted Date: December 8th, 2020

DOI: https://doi.org/10.21203/rs.3.rs-107943/v1

License: (9) This work is licensed under a Creative Commons Attribution 4.0 International License. Read Full License

Version of Record: A version of this preprint was published at BMC Bioinformatics on December 1st, 2021. See the published version at https://doi.org/10.1186/s12859-021-04503-y. 
Five crucial prognostic-related autophagy genes stratified female breast cancer

\section{patients aged 40-60 years}

Xiaolong Li\# ${ }^{1}$, Hengchao Zhang\# ${ }^{1}$, Jingjing Liu ${ }^{1}$, Ping Li ${ }^{1}$, Yi Sun*1

1 Surgical department of breast thyroid surgery, Xuchang Central Hospital, 461600,China

\# Co-first author:Xiaolong Li and Hengchao Zhang

* Corresponding author: Yi Sun

Address:No.30 Huatuo Road,Weidu District,461600,Xu Chang ,Henan Province, China

TEL: 0374-3353521

Email:

Xiaolong Li: xiaolonglirjk@163.com

Hengchao Zhang: tj08-6021@163.com

Jingjing Liu: jingjingliucsk@163.com

Ping Li: 13613743117@139.com

Yi Sun: sunyi19700420@163.com

The hospital website: http://www.xcszxyy.cn/ 


\section{Abstract}

Background : Autophagy is closely related to the progression of breast cancer. The aim at this study is to establish a prognostic-related model comprised of hub autophagy-genes (AGs ) to assess patient prognosis . Simultaneously, the model can guide clinicians to make up individualized strategies and stratify patients aged 40-60 years based on risk level. Methods : The hub AGs were identified with univariate COX regression and LASSO regression. The functions and alterations of these selected AGs were analyzed as well . Moreover, the multivariate COX regression and correlation analysis between hub AGs and clinicopathological parameters were done . Results : Totally, 33 prognostic-related AGs were obtained from the univariate COX regression $(\mathrm{P}<0.05)$. SERPINA1 , HSPA8 , HSPB8, MAP1LC3A, and DIRAS3 were identified to constitute the prognostic model by the LASSO regression . The survival curve of patients in high-risk and in low-risk group was statistically significant $(\mathrm{P}<0.05)$. The 3-year and 5-year ROC displayed that their AUC value reached 0.762 and 0.825 , respectively . Stage and risk score were independent risk factors relevant about prognosis . RB1CC1, RPS6KB1, and BIRC6 were identified as the most predominant mutant genes. It was found that AGs were mainly involved in regulating the endopeptidases synthesis and played important roles in ErbB signal pathway. SERPIN1, risk score were closely related to stage $(\mathrm{P}<0.05)$; HSPA8, risk score were closely related to $\mathrm{T}$ stag $(\mathrm{P}<0.05)$; HSPB8 was closely related to $\mathrm{N}$ stag $(\mathrm{P}<0.05)$. Conclusions : Our prognostic model had relatively robust predictive ability on prognosis for patients aged 40-60 years . If stage was added into 
the prognostic model, the predictive ability would be more powerful .

Key words Autophagy genes ; SERPINA1 ; HSPA8 ; HSPB8 ; MAP1LC3A ;

DIRAS3

\section{Introduction}

Autophagy is a natural phenomenon that regulates cell metabolism inside. Under normal physiological conditions, immune cell autophagy process could eliminate senile organelles and abnormal long-lived proteins in the body, which will be conducive to maintaining immune cell homeostasis. However, a stress stimuli, could result in cell autophagy working in preventing the accumulation of toxic or carcinogenic damage proteins and organelles, and inhibiting simultaneously cell cancelation. If it was occurred dysfunctional autophagy process, tumor could be coming soon[1]. Therefore, during oncogenesis process, autophagy has double function with promotion and suppression.It has been implicated that tumorigenesis is closely related to cell autophagy[2].

Breast Cancer $(\mathrm{BC})$ is one of the most common malignancies for women. The 5-year survival rate of patients is low and it is easy to turn out especially on middle-aged 40-60 years women.Given it is prone to distant metastases, early diagnosis is of great significance of the prognosis of $\mathrm{BC}$ patients[3]. So far, conventional clinical evaluation means, such as TNM stag and pathological classification, are tough to precisely speculate patients prognosis in fact and offer clinical doctors more accurate treatment choices. In our study, analyzing the RNA-sequence data onto the AGs from female BC patients at a high incidence age, a prognostic model composed of several 
signatures was conceived, more accurately dividing BC patients into high-risk and low-risk groups, which will benefit to clinicians to target Patients in various risk levels and to adopt corresponding individualized strategies.

\section{Methods}

\section{Download data}

The Illumina HiSeq data and clinical data of female BC patients, whose ages were 40-60 years and whose pathological types were ductal and lobular tumors, were downloaded from The Cancer Genome Atlas (TCGA, https://www.cancer.gov/about-nci/organization/ccg/research/structural-genomics/tcga,) and Gene Expression Omnibus (GEO, https://www.ncbi.nlm.nih.gov/gds/) databases.

Filter out prognostic AGs and adventurous clinical factors for constructing the model

The univariate and multivariate COX regression (Cox proportional-hazards model) was performed to screen prognostic AGs by R software package"survival"[4]., while the Least Absolute Shrinkage and Selection Operator ( LASSO regression) was to construct the model of autophagic gene prognosis and to calculate patients riskscore through R software package"glmnet”..By means of including clinical and pathological factors, the multivariate COX regression could filter out independent risk factors, which were jointly incorporated into the model construction.

\section{The various indexes evaluated the practicality of this predictive model}

The performance of the AGs model was assessed by plotting survival curves, risk curves, Receiver Operating Characteristic Curve (ROC), calibration curves.We 
analyzed the main functions of prognostic-related AGs by gene ontology (GO) and Kyoto Encyclopedia of Genes and Genomes (KEGG). Gene alterations were detected in cBioportal database(http://www.cbioportal.org/). Simultaneously, the correlation analysis was done between hub AGs and clinical factors.

\section{Externally independent validation}

The generalization ability of our risk score model was verified in GEO[5]. The Human Protein Atlas (HPA, https://www.proteinatlas.org/) was used for validating the hub prognostic AGs on a protein level.

\section{Statistics}

$\mathrm{R}$ software 3.4.2 was used for statistic analysis and drawing pictures.It can perform Kaplan-Meier, LASSO and multivariate COX regression,ROC,calibration curves,as well as correlation analysis. $\mathrm{P}<0.05$ was regarded as existed significant difference statistically.

\section{Results}

\section{Screening prognostic- related AGs by univariate COX regression}

To download the RNA-sequence data and survival data onto 501 female $\mathrm{BC}$ patients whose ages were 40-60 years old from TCGA database, the expression data of the whole 194 AGs were extracted,in terms of AGs list in Human Autophagy Database: HADb (http://autophagy.lu/). The univariate cox regression analysis was performed on $501 \mathrm{BC}$ samples, and 33 prognostic-related AGs were obtained, in which 23 up-regulatedand ones and 10 down-regulated ones (Figure 1A). Simultaneously, there were significant differences in these genes expressions of healthy women and female 
BC patients $(\mathrm{P}<0.05)$ (Figure 1B).

\section{The five hub AGs identified by LASSO regression}

The five AGs were selected from 33 prognostic-related AGs by LASSO regression, whose risk score were calculated based on their respective correlation coefficients for risk stratification of BC prognosis (Figure 1C). At lambda.1se ,there were five AGs screened, Serpin family A member 1 (SERPINA1), Heat shock protein family A (Hsp70) member 8 (HSPA8), Heat shock protein family B (small) member 8 (HSPB8), Microtubule associated protein 1 light chain 3 alpha (MAP1LC3A), and DIRAS family GTPase 3 (DIRAS3) (Figure 1D).Formula:Riskscore = SERPINA1 expression levels* $(-0.107130438454055)+$ HSPA8 expression levels* $0.0342643673417569+$ HSPB8 expression levels* 0.0320959903903294 + MAP1LC3A expression levels * $(-0.0206809753743301)+$ DIRAS3 expression levels * (-0.0415014293356393). On the protein level, we validated these hub genes expressions of normal tissues and tumor tissues through HPA database (supplementary file) .HSPA8 and HSPB8 expressed higher in tumor tissues than that in normal tissues, while SERPINA1 and DIRAS3 expressed higher in normal tissues against tumor tissues. MAP1LC3A weakly expressed in tumor and normal tissues (Figure S1).

\section{The differentiated survival curve depended on five-AGs-riskscore between the}

\section{high-risk group and the low-risk group}

At the risk score cut-off value -0.2 , all patients were entered into high risk group ( $>$ $-0.2)$ and low risk group $(<=-0.2)$. The difference in survival curves between the high-risk group and the low-risk group was statistically significant $(\mathrm{P}=6.639 \mathrm{e}-06)$. 
The survival rate of patients with high risk score was significantly lower than that of patients with low risk score (Figure 1E). SERPINA1, MAP1LC3A, DIRAS3 were down-regulated in high risk group and up-regulated in low risk group, which were potentially tumor suppressor genes; HSPA8, HSPB8 were up-regulated in high risk group and down-regulated in low risk group, which were probably promoting oncogenes. The number of died patients in high risk group was significantly more than that in low risk group (Figure 1F).

\section{Identification of the independent risk prognostic factors}

The univariate and multivariate COX regression analysis on various clinical pathological parameters was done, such as age, stage, $\mathrm{T}, \mathrm{M}, \mathrm{N}$ and risk score, in order to screen independent risk factors affecting prognosis. Univariate $\mathrm{COX}$ regression analysis suggested that stage, $\mathrm{T}, \mathrm{N}$, and risk score were risk factors affecting the prognosis of $\mathrm{BC}$ patients $(\mathrm{P}<0.05, \mathrm{HR}>1)$ (Figure $2 \mathrm{~A}$ ), while multivariate $\mathrm{COX}$ regression suggested that stage and risk score were independent risk factors $(\mathrm{P}<0.05$, $\mathrm{HR}>1$ ) (Figure 2B). The AUC(Area Under the Curve) values of risk score reached to 0.762 in three years and 0.825 in five years. The AUC of stage alone in three years also reached to 0.758. However,for five year, the AUC value was 0.682. The AUC value of stage combined with risk score was 0.844 in three years and 0.832 in five years (Figure 2C ,Figure 2D). It was implicated that stage and risk score was more accurate than alone risk score on predictive ability.

5.Correlation analysis between the five hub AGs expression and clinicopathological parameters 
We attempted to discover whether the five key AGs and their risk score were correlated with these clinicopathological factors. SERPIN1, risk score and stage were closely related $(\mathrm{t}=9.268, \mathrm{P}=0.026 ; \mathrm{t}=11.79, \mathrm{P}=0.008)$; HSPA8, risk score and $\mathrm{T}$ stag is closely related $(\mathrm{t}=17.535, \mathrm{P}=0.484 \mathrm{e}-04 ; \mathrm{t}=8.185, \mathrm{P}=0.042)$; HSPB8 is closely related to $\mathrm{N} \operatorname{stag}(\mathrm{t}=8.136, \mathrm{P}=0.043)$ (Table 1,Figure 2E).

\section{The performance of clinical prognostic model based on five hub AGs}

Double independent prognostic risk factors, stage and risk score, were included in an established nomogram (Figure 2F). C-index reached 0.745 (95\% CI, 0.709- 0.778). At the same time, we plotted the calibration curve and further evaluated the accuracy of our model. Its results were showed that there was no statistically significant difference between model predicted value and the true one (Figure 2G,Figure $2 \mathrm{H}$ ). The multi-factor ROC illuminated that risk score has the most robust predictive ability for $\mathrm{BC}$ prognosis among all clinicopathological factors.

\section{The function analysis of prognostic-related AGs}

The prognosis-related $\mathrm{AGs}$ of $\mathrm{BC}$ selected by univariate $\mathrm{COX}$ regression were performed $\mathrm{GO}$ and $\mathrm{KEGG}$ function enrichment analysis. It was found that up-regulated AGs were mainly involved in the regulation of endopeptidases in vivo (Figure 3A), while down-regulated AGs were mainly involved in adjusting ErbB signaling (Figure 3B). We have also taken the cBioPortal database (https://www.cbioportal.org/) of BC patients (METABRIC, Nature 2012 \& Nat Commun 2016) with mutations and putative copy-number changing from DNAcopy to verify the genetic alteration. As a consequence, $815(38 \%)$ s of 2173 
patients/samples had occurred genetic changes, in which RB1CC1 (12\%), RPS6KB1 (11\%), and BIRC6 (6\%) had the most genetic variety (Figure 3C). There was a significant discrepancy in survival rate of for the genetically altered group and the unaltered group $(\mathrm{P}=5.242 \mathrm{e}-3)$, and the prognosis of patients in the genetically modified group was worse (Figure 3D).

\section{The external validation of the AGs prognostic models on GEO database}

To confirm the practicality of the five-hub-AGs prognostic model, we downloaded GSE96058, with platform GPL11154, Series Matrix File (s) and RNA sequence data from GEO.Randomly select 200 patients aged 40-60 years from 3273 samples, and calculate five-AGs-riskscore. At cut-off value risk score -0.2 , patients were divided into a high-risk group and a low-risk group. The evident difference was existed on both groups, and the survival rate of high-risk patients was significantly lower than that of low-risk groups $(\mathrm{P}=4.383 \mathrm{e}-04)$ (Figure 3E). The ROC curve of the verification group showed that risk score has relatively good generalization ability (Figure 3F).

\section{Discussion}

Autophagy is a complex immune process regulated genetically, in which autophagy-related genes are involved. It can supervise genome alteration and to impair genomic instability[6]. Carcinogenesis is subjective to genetic mutations. The role of AGs in tumorigenesis and progression is extremely complicated and its function is likely to be multiple in diverse stages of cancer progression[7]. In the early stage, autophagy might preserve cell survival through cellular catabolism. The 
expression of AGs might hinder cells proliferation with cancer-associated mutation tendency, preventing tumorigenesis by promoting senescence. However, once the tumor has exhibited, cancer cells can protect themselves by autophagy. During the late stage of tumorigenesis, autophagy can increase oxidative stress, thereby to facilitate genomic instability and malignant transformation.

The 5-year survival rate of $\mathrm{BC}$ patients is about $26 \%$, nearly $12 \%$ of whom eventually develops to metastatic disease, meaning cancer cell disseminate to other organs from breast[8]. with a median survival time of 18-30 months, it would induce poor prognosis and high mortality $[9,10]$. To date,some scholars have contributed to the relationship between $\mathrm{BC}$ and autophagy. Vega-Rubín-de-CelisS et al.[11] have explored the regulated mechanism of HER2 to autophagy in vita, and the impacts of genetic and pharmacological strategies on HER2-driven BC proliferation enhancing autophagy in vivo. It was found that HER2 mediated inhibition for Beclin 1 autophagy might be beneficial to HER2-mediated tumorigenesis, and the strategies blocking HER2 / Beclin 1 binding or increased autophagy were promising on curing HER2-positive BC patients. Timothy Marsh et al.[12] temporarily removed essential autophagy regulators during cancer progression by BC model. Although AGs ablation intensively weakened the growth of primary breast tumors, impaired autophagy would promote spontaneous metastasis and causes metastatic tumor cells apparently to be large ones. Their research results suggested that NBR1 autophagy-dependent administration was a crucial determinant of the metastatic process. Yeo SK et al.[13] have verified whether macrophage autophagy inhibition was effective in 
BRCA1-deficient breast tumors. By the K14-Cre transgene, they created mice with conditionally deleted basic AGs, Rb1cc1, Brca1 and Trp53. Finally,they found that autophagy suppression could increase profits of dimethyldibase treating BRCA1-deficient BC. Walker A et al.[14] discovered the protective mechanism of BC cells under condition of glucose deprivation. Nrf2 signaling played a vital role in protecting $\mathrm{BC}$ cells during glucose deprivation-induced autophagy through its antioxidant active. Ji Y et al.[15] examined the effects of NVP-BEZ235 to viability, apoptosis and autophagy in BC cell lines. They also tested how NVP-BEZ235 made an influence on the expression of p-AKT, p-mTOR and p-70S6K in the pathway PI3K / AKT / mTOR. The results showed us that NVP-BEZ235 significantly suppressed BC cells proliferation and induced apoptosis and autophagy in MCF-7 cells. Lozy F, et al.[16] found that low-exressed BECN1 was associated with ERBB2 overexpression in $\mathrm{BC}$, which suggested that BECN1 deletion and ERBB2 overexpression might functionally interact during breast tumorigenesis. ERBB2 overexpression repressed autophagy respond to stress on mouse mammary glands and Human BC cells. ERBB2-driven breast tumorigenesis was closely correlated with functional autophagy inhibition. Hamurcu Z, et al.[17] detected the role of FOXM1 in regulating autophagy in TNBC (triple negative BC) cells. FOXM1 expression was up-regulated during the process of autophagy induction. They found that repressing FOXM1 could inhibit autophagy induced by starvation and rapamycin and the expression of major autophagy regulators, LC3 and Beclin-1.

Notably, despite many studies have explored the role of autophagy in tumorigenesis, 
most work has focused on analyzing single autophagy gene in one or two cell lines or animal models. Few people have concerned about the meaning of prognostic datasets with high-throughput sequence expression Profiles in BC. With the coming of high-throughput "omics" data, it is now likely to study expression patterns of global AGs and their involvement in predicting BC outcomes.

In our study, prognostic-related AGs were analyzed by high-throughput expression profile. Eventually, SERPINA1, HSPA8, HSPB8, MAP1LC3A and DIRAS3 were identified to be hub AGs related with prognosis and to calculate the patients' risk score. When a patients' risk score was more than -0.2 , the patient would be regarded to be at high risk. When his or her risk score was less than -0.2 , we consider the patient to be at low risk. Besides it, we have compared the survival rates of patients at high risk and patients at low risk, which existed significant differences. The AUC value of the ROC for evaluating the 5-year survival rate of $\mathrm{BC}$ patients was 0.825 . If another independent risk factor, stage,was added into our model, the AUC value would reach to 0.832 .

Futhermore, the relationship between these five AGs and BC had been studied by other scholars as well. Chan HJ et al.[18] discovered that ER was constitutively activated, resulting in binding with SERPINA1 gene and up-regulation of SERPINA1 expression. High expression of SERPINA1 might herald better clinical outcomes of $\mathrm{ER}+$ and ER + / HER2 + patients. Yu B et al.[19] believe that HSPA8 (heat shock protein 70) was a target for anti-cancer therapy. Overexpression of HSPA8 was usually observed in BC. Inhibition of HSPA8 expression could induce apoptosis of 
BC cells. Piccolella $M$ et al.[20] found that HSPB8 was highly expressed in triple-positive hormone-sensitive $\mathrm{BC}$ cells (MCF-7) and was involved in regulating cell cycle and cell migration. Othman EQet al.[21] uncovered that, compared with normal tissues, a large number of BC tissues expressed the MAP1LC3A protein with strong immunoreactivity. It was indicated that MAP1LC3A was likely to play an indispensable role in the progression of BC. Nowak EM et al.[22] revealed that DIRAS3 could regulate cell cycle and impair the growth and movement of cancer cells, all of which might be indirectly depended on the interaction with STAT3. The down-regulation of DIRAS3 expression was related to the progression of $\mathrm{BC}$, while the restoration of DIRAS3 expression could restain cell proliferation and invasiveness. We also performed the functional analysis of prognostic-related AGs and found that they were mainly engaged in the regulation of endopeptidase and ERBB2 signaling pathways. Sisinni L et al.[23] focused on endoplasmic reticulum stress and the relationship between apoptosis and autophagy in human BC. They thought that endoplasmic reticulum stress resulted in levels and activities alteration of key regulators about cell survival and autophagy, promoting to increase endopeptidase synthesis. Moreover,the ERBB2 signaling pathway was a critical pathway to regulating $\mathrm{BC}$ autophagy[16].

We also compared other $\mathrm{BC}$ prediction models to ours. It was implicated that our model was simpler and easier to operate than theirs, and the AUC value of reflecting predictive ability was relatively higher. Shen $\mathrm{Y}$ et al.[24] investigated that 11-lncRNA signature was a novel and significant prognostic element independent among multiple 
clinical and pathological parameters. The TIMER database showed that the the 11-IncRNA prognostic characteristic was relevant to the infiltration of immune cell subtypes in $\mathrm{BC}$. Wu Y et al.[25] identified vital pathways revealing potential molecular mechanisms and core genes probably used as BC biomarkers by means of WGCNA( Weighted Correlation Network Analysis). The three central genes, CBR3, SF3B6 and RHPN1,might play important roles in malignancy cancer transformation. Gu Y et al.[26] mentioned that eight AGs, BCL2, BIRC5, EIF4EBP1, ERO1L, FOS, GAPDH, ITPR1, and VEGFA, were ought to be designated as prognostic markers for autophagy-associated BC. Lin QG et al.[27] initially found that 27 ARGs (autophagy-related genes) were evidently associated with overall survival rate of BC. The prognostic-related ARGs signature established by the Cox regression consisted of 12 ARGs with a 5-year survival rate AUC 0.742.

Of course, our autophagy-related risk score model is not perfect. The expression of these five hub genes is supposed to be verified in other independent datasets, and the practicality of this predictive model should be detected through feedbacks from clinical applications. In addition, researches on the function of AGs are particularly meaningful. For instance, how autophagy deletion or autophagy inhibition promotes the gene expansion and growth of $\mathrm{BC}$ cells, and the interaction mechanism between its functions remains to be investigated, which has great significance for the treatment and prognosis of $\mathrm{BC}$ patients. The functional status of autophagy and its pharmacological manipulation of $\mathrm{BC}$ treatment will become novel targeted treatment options. 


\section{Conclusions}

we have constructed a prognostic model for predicting the survival rate of BC patients composed of five essential AGs, attempting to provide guidance of clinicians on making up personalized treatment strategies corresponded to different risk level patients. The autophagy signature represents a promising biomarker for assessing the overall survival rates of $\mathrm{BC}$ patients.

\section{Ethics statement}

The informed consent was obtained from all subjects. Our study has been approved by medical ethics committee of Xuchang Central Hospital.Number:2020-002

\section{Methods statement}

All methods were carried out in accordance with relevant guidelines and regulations.

\section{Consent for publication}

All authors have seen the manuscript and approved of publication.

\section{Conflict of interest statement}

The authors have declared that there is no interest conflict .

\section{Data availability statement}

The data that support the findings of this study are available in TCGA and GEO at [https://www.cancer.gov/about-nci/organization/ccg/research/structural-genomics/tcga] ,[https://www.ncbi.nlm.nih.gov/geo/query/acc.cgi?acc=GSE96058],reference number [GSE96058]. These data were derived from the following resources available in the public domain: The cancer genome atlas and Gene Expression Omnibus.

\section{Funding statement}


No available funding supported this research.

\section{Author Contributions}

Conceive and design the article: XL L and HC ZH. Collect the patients information:

JJ L. Statistics data: P L. Write the paper: XL L.Surpervision:Y S.

\section{Acknowledgements}

Thank you for RNA sequence data provided with TCGA database and GEO database.

\section{References}

[1] Mowers EE, Sharifi MN, Macleod KF,Functions of autophagy in the tumor microenvironment and cancer metastasis[J].FEBS J. 2018 May;285(10):1751-1766.

[2] Kimmelman AC, White E.Autophagy and Tumor Metabolism[J].Cell Metab. 2017 May 2;25(5):1037-1043.

[3] Woolston C.BC[J].Nature. 2015 Nov 19;527(7578):S101.

[4] Hongzhe Li, Jiang Gui, Partial Cox regression analysis for high-dimensional microarray gene expression data[J]. Bioinformatics, Volume 20, Issue suppl_1, 4 August 2004, Pages i208-i215.

[5] Kawaguchi T, Yan L, Qi Q, et al. Novel MicroRNA-based risk score identified by integrated analyses to predict metastasis and poor prognosis in breast cancer $[\mathrm{J}]$. Annals of surgical oncology, 2018, 25(13): 4037-4046.

[6] Levine B, Kroemer G,Biological Functions of Autophagy Genes: A Disease Perspective [J]. Cell. 2019 Jan 10;176(1-2):11-42.

[7] Yanyan H, Shujun F, Tao Q, et al. Role of autophagy in BC and BC stem cells (Review)[J]. International Journal of Oncology, 2018. Apr;52(4):1057-1070

[8] Peart O.Metastatic BC[J].Radiol Technol. 2017 might;88(5):519M-539M.

[9] Walker DK, Whitehead L,Breast surgery for metastatic $\mathrm{BC}[\mathrm{J}]$.Br J Community Nurs. 2018 Jul 2;23(7):334-335.

[10]Narod SA, Giannakeas V, Sopik V,Time to death in BC patients as an indicator of treatment response[J].BC Res Treat. 2018 Dec;172(3):659-669.

[11] Vega-Rubín-de-Celis, Silvia, Zou, et.al. Increased autophagy blocks HER2-mediated breast tumorigenesis[J]. Proc Natl Acad Sci U S A. 2018 Apr 17;115(16):4176-4181

[12]Marsh,Kenific,Suresh,etal.Autophagic degradation of NBR1 restricts metastatic o utgrowth during mammary tumor progression[J].Dev Cell. 2020 Feb 12. 
[13]Yeo SK, Paul R, Haas M, et al. Improved efficacy of mitochondrial disrupting agents upon inhibition of autophagy in a mouse model of BRCA1-deficient BC.[J]. Autophagy, 2018.14(7):1214-1225

[14]Walker A, Singh A, Tully E, et al.Nrf2 signaling and autophagy are complementary in protecting $\mathrm{BC}$ cells during glucose deprivation[J].Free Radic Biol Med. 2018 might 20;120:407-413.

[15]Ji Y, Di W, Yang Q,et al. Inhibition of Autophagy Increases Proliferation Inhibition and Apoptosis Induced by the PI3K/mTOR Inhibitor NVP-BEZ235 in Breast Cancer Cells.[J].Clin Lab. 2015;61(8):1043-51.

[16]Lozy F, Cai-McRae X, Teplova I,et al.ERBB2 overexpression suppresses stress-induced autophagy and renders ERBB2-induced mammary tumorigenesis independent of monoallelic Becn1 loss[J].Autophagy. 2014 Apr;10(4):662-76.

[17] Hamurcu Z, Delibaşı N, Nalbantoglu U, et al.FOXM1 plays a role in autophagy by transcriptionally regulating Beclin-1 and LC3 genes in human triple-negative BC cells[J].J Mol Med (Berl). 2019 Apr;97(4):491-508.

[18]Hei Jason Chan, Haiqing Li, Zheng Liu,et al. SERPINA1 is a direct estrogen receptor target gene and a predictor of survival in $\mathrm{BC}$ patients[J]. Oncotarget, 2015, 6(28):25815-25827.

[19] Yu B, Yang H, Zhang X, Li H.Visualizing and Quantifying the Effect of the Inhibition of HSP70 on Breast Cancer Cells Based on Laser Scanning Microscopy.Technol Cancer Res Treat. 2018 Jan 1;17:1533033818785274.

[20]Piccolella M, Crippa V,, Cristofani R,et al.The small heat shock protein B8 (HSPB8) modulates proliferation and migration of BC cells[J].Oncotarget. 2017 Feb 7;8(6):10400-10415.

[21]Ekhlas Qaid Gazem Othman, Gurjeet Kaur, Ahmad Faisal Mutee,et al.Immunohistochemical expression of MAP1LC3A and MAP1LC3B protein in breast carcinoma tissues[J]. Journal of Clinical Laboratory Analysis, 2009, 23(4):249-258.

[22]Nowak E M, Poczęta M, Bieg D, et al. DNA methyltransferase inhibitors influence on the DIRAS3 and STAT3 expression and in vitro migration of ovarian and breast cancer cells[J].Ginekol Pol. 2017;88(10):543-551.

[23] Sisinni, Pietrafesa, Lepore,etal.Endoplasmic reticulum stress and unfolded protein response in breast cancer: The balance between apoptosis and autophagy and its role in drug resistance[J].Int J Mol Sci. 2019 Feb 16;20(4).

[24] Shen Y, Peng X, Shen C,Identification and validation of immune-related lncRNA prognostic signature for BC[J].Genomics. 2020 Feb 19.

[25] Wu Y, Liu F, Luo S, et al.Co-expression of key gene modules and pathways of human BC cell lines[J]. Biosci Rep. 2019 Jul $18 ; 39(7)$. 
[26] Gu Y, Li P, Peng F, et al.Autophagy-related prognostic signature for $\mathrm{BC}[\mathrm{J}] . \mathrm{Mol}$ Carcinog. 2016 Mar;55(3):292-9.

[27]Lin QG, Liu W, Mo $\quad$ YZ,et al. Development of prognostic index based on autophagy-related genes analysis in br east cancer[J].Aging (Albany NY). 2020 Jan 22;12(2):1366-1376.

\section{Additional File}

Supplementary file.doc

Figure S1 The expression of the five hub prognostic AGs on the protein level

The database provided immunohistochemistry (IHC) results using a tissue microarray (TMA)-based analysis of the corresponding proteins in PC patients and adjacent normal tissues. IHC staining for each gene was done using the same antibodies in tumor tissues as in normal tissues. However, the estimation of protein expression could not be performed.

\section{Legend}

Table 1 The correlation coefficients between key autophagy genes and clinicopathological factors $(\mathbf{r} 2, \mathrm{P})$

Figure 1 Establish the prognostic-related riskscore model comprised of five mRNA genes . (A) Univariate COX regression analysis was to screen potential autophagy genes related to prognosis.red scale:upregulated genes;green scale:downregulated genes. (B) Validate selected autophagy genes expression in normal group(N) and tumor group(T). (C) LASSO regression filtering out the most representive five core autophagy genes. (D) 10-fold cross validation was to identify the minimal lambda and its 1-standard error away(1-se) with the least core autophagy genes. Left vertical dashed line:at minimal lambda 0.01053932;Right vertical dashed line: at 1-se minimal lambda 0.03532362.(E)The survival rate comparison between high riskscore and low risk riskscore patients.(F)The riskplot for high riskscore and low riskscore patients.Vertical dashed line is the boundary of both group patients.

Figure 2 Clinical and pathological factors involved in constructing the riskscore model.(A) Univariate COX regression.(B) Multivariate COX regression.red scale:Hazard ratio>1;green scale:Hazard ratio<1.(C) ROC for 3-years survival rate. (D) ROC for 5-years survival rate.(E) The correlation analysis of critical autophagy genes with clinical factors. (F) Nomogram built by all high risk factors.(G) Calibration curve for 3-years prediction.(H) Calibaration curve for 5-years prediction.

Figure 3 The genes function enrichment analysis and external validation. (A) GO cricle of genes function. (B) KEGG circle of pathway enrichment analysis. (C) Oncoprint reflecting autophagy genes alteration. (D) Sruvival differentiation among altered group and unaltered group.(E) Survial differentiation between high and low riskscore patients in GEO set.(F) ROC accessment in GEO independent validation cohort. 
Figures
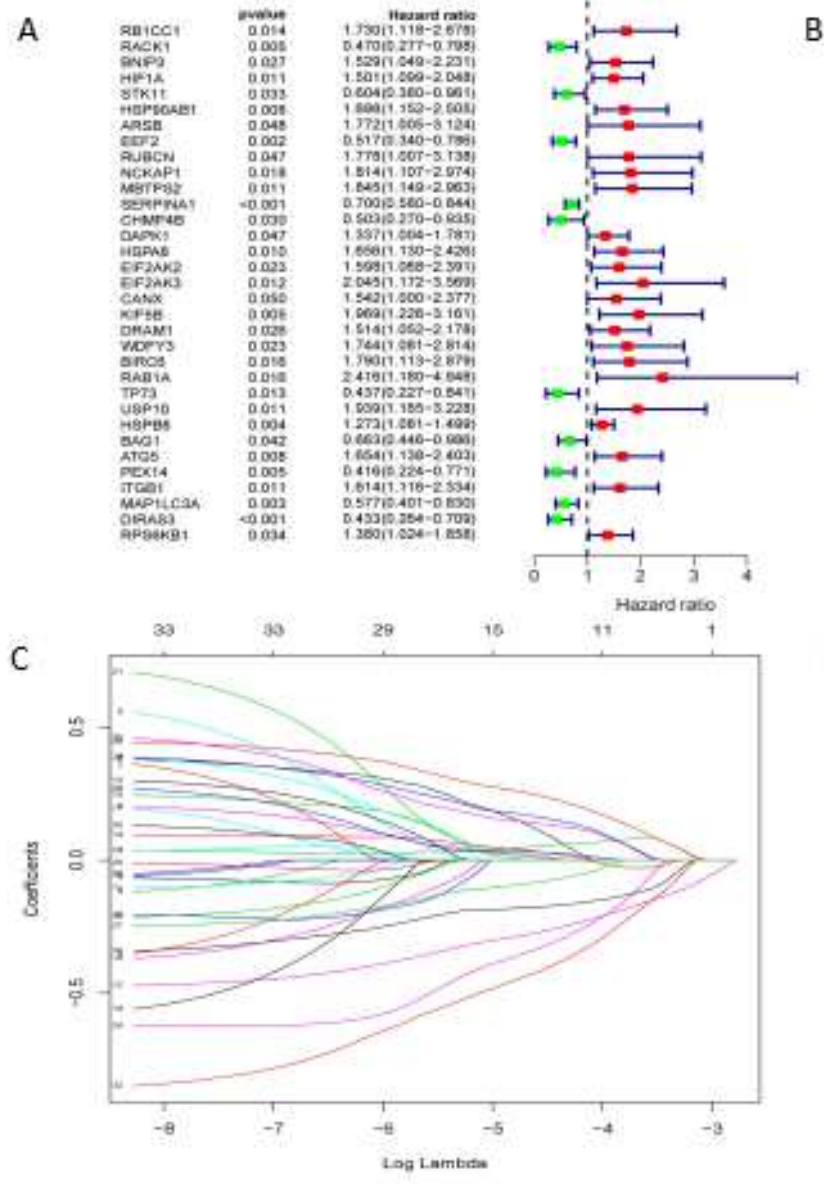

E

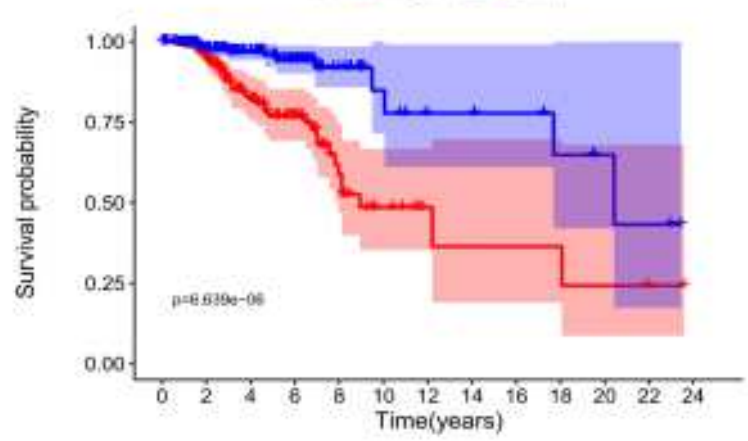

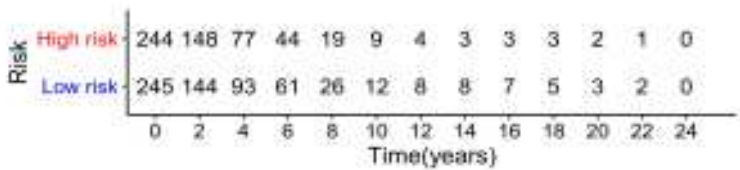

B

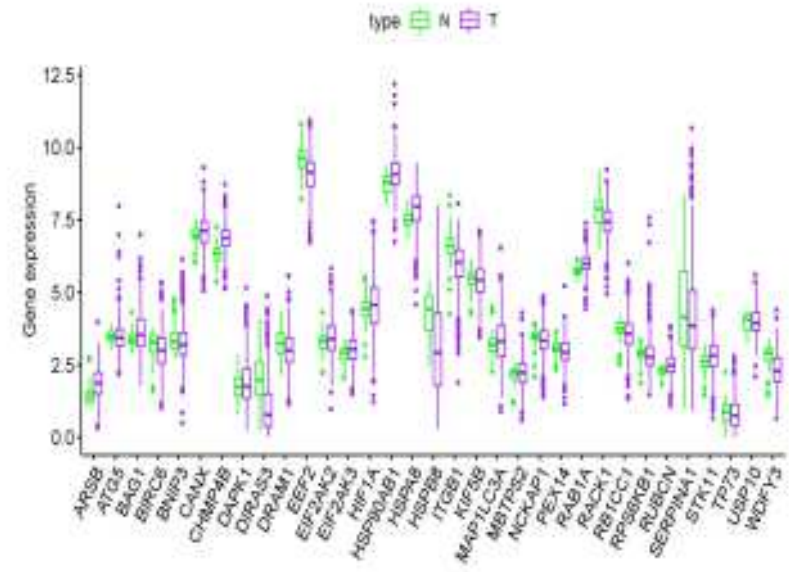

D

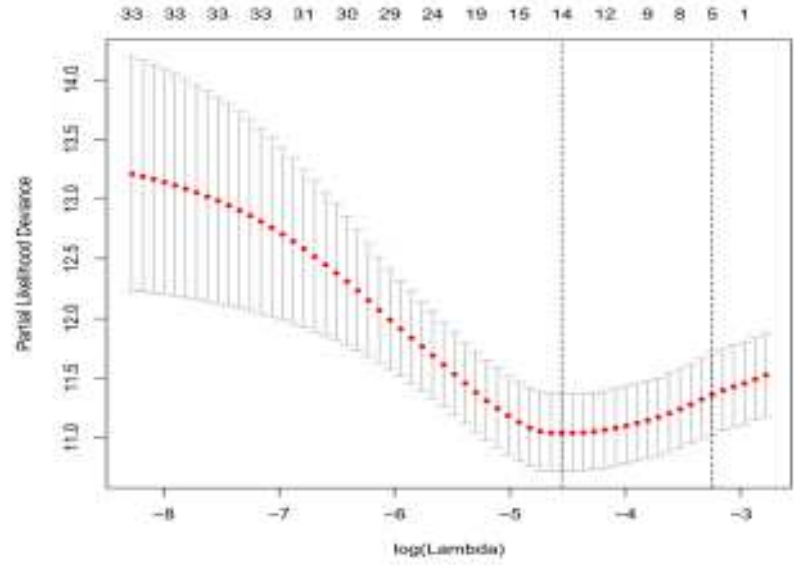

$\mathrm{F}$
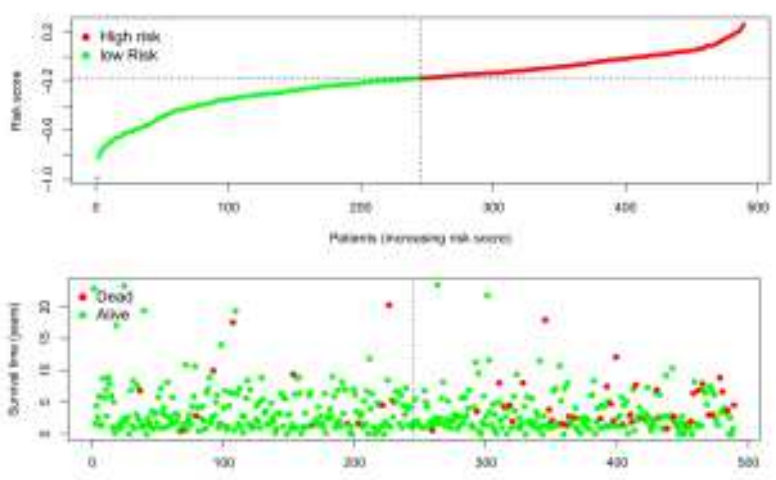

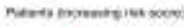

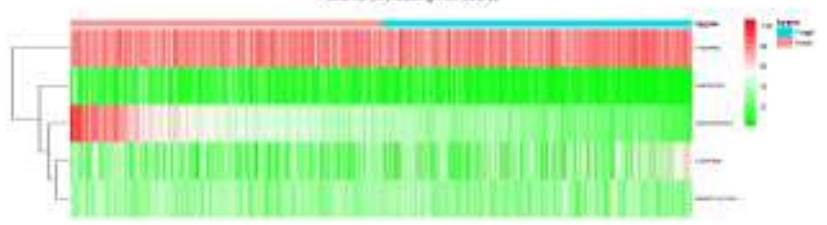

\section{Figure 1}

Establish the prognostic-related riskscore model comprised of five mRNA genes . (A) Univariate COX regression analysis was to screen potential autophagy genes related to prognosis.red scale:upregulated genes;green scale:downregulated genes. (B) Validate selected autophagy genes expression in normal 
group $(\mathrm{N})$ and tumor group $(\mathrm{T})$. (C) LASSO regression filtering out the most representive five core autophagy genes. (D) 10-fold cross validation was to identify the minimal lambda and its 1-standard error away(1-se) with the least core autophagy genes. Left vertical dashed line:at minimal lambda 0.01053932;Right vertical dashed line: at 1-se minimal lambda 0.03532362.(E)The survival rate comparison between high riskscore and low risk riskscore patients. $(F)$ The riskplot for high riskscore and low riskscore patients.Vertical dashed line is the boundary of both group patients.

A
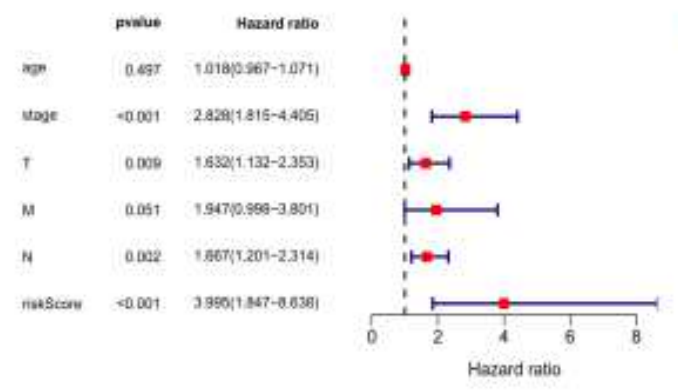

c

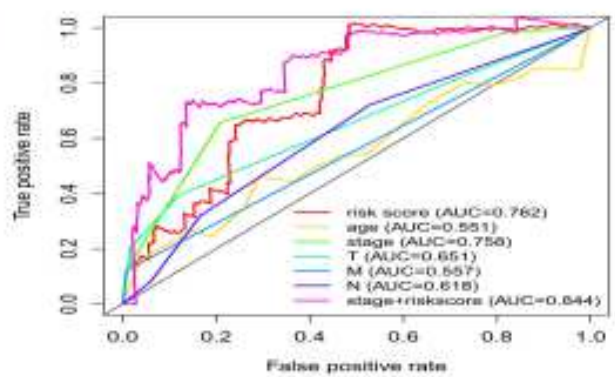

E
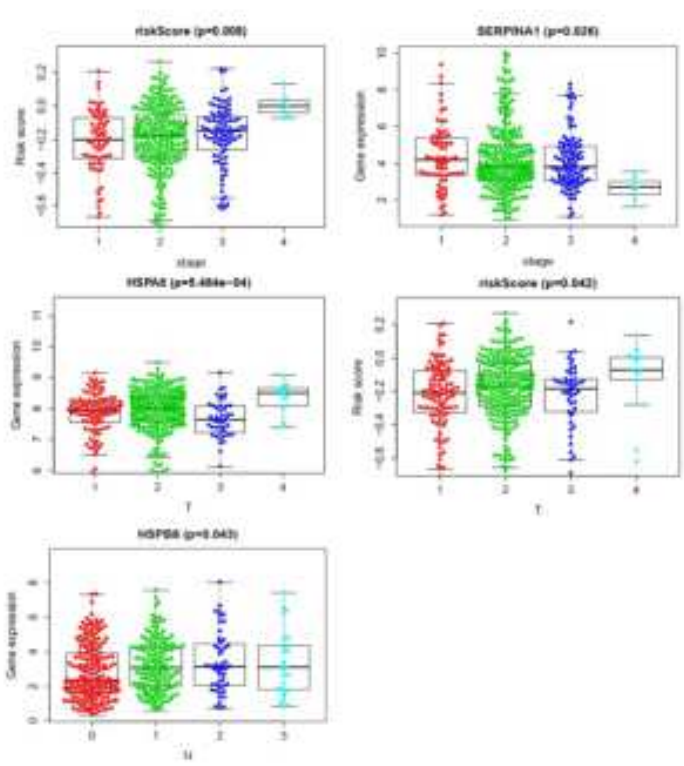

G

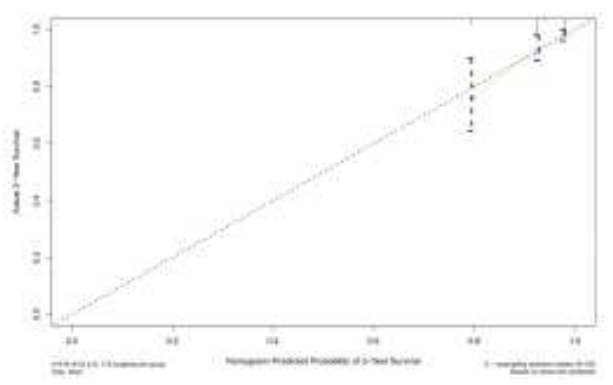

B

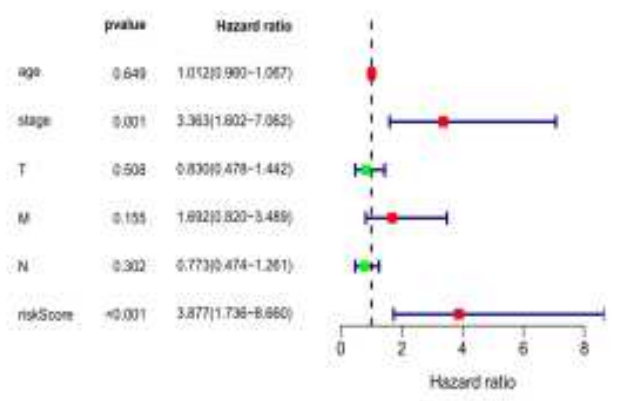

D

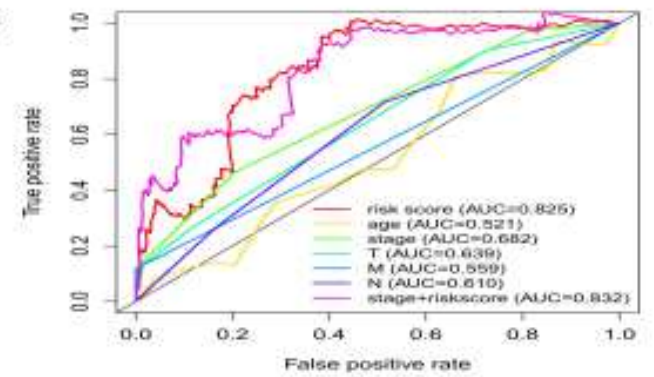

F

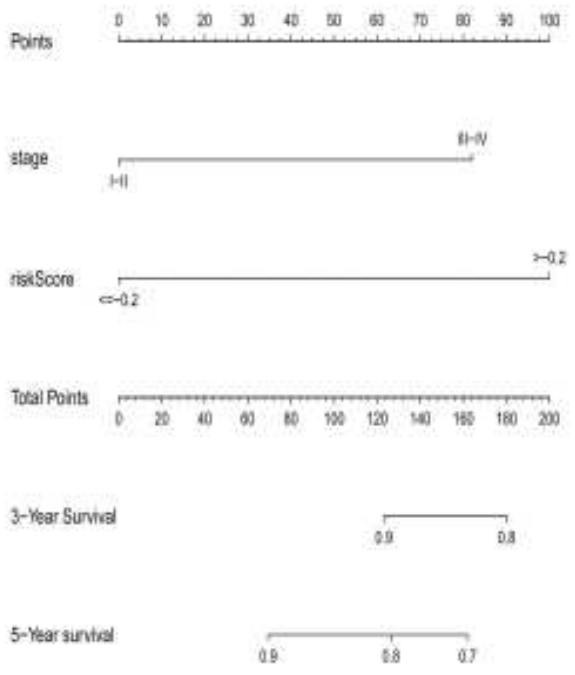

H

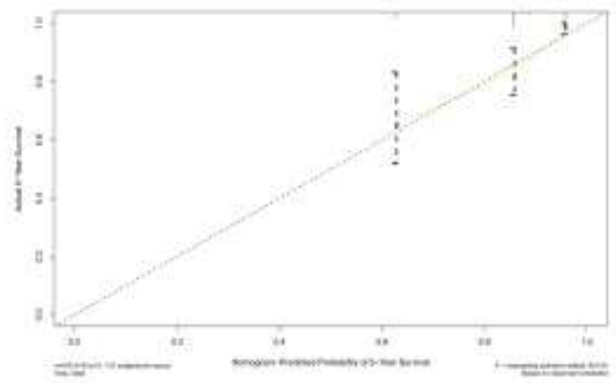

Figure 2 
Clinical and pathological factors involved in constructing the riskscore model.(A) Univariate COX regression.(B) Multivariate COX regression.red scale:Hazard ratio $>1$; green scale:Hazard ratio<1.(C) ROC for 3-years survival rate. (D) ROC for 5-years survival rate.(E) The correlation analysis of critical autophagy genes with clinical factors. (F) Nomogram built by all high risk factors.(G) Calibration curve for 3-years prediction.(H) Calibaration curve for 5-years prediction.

A
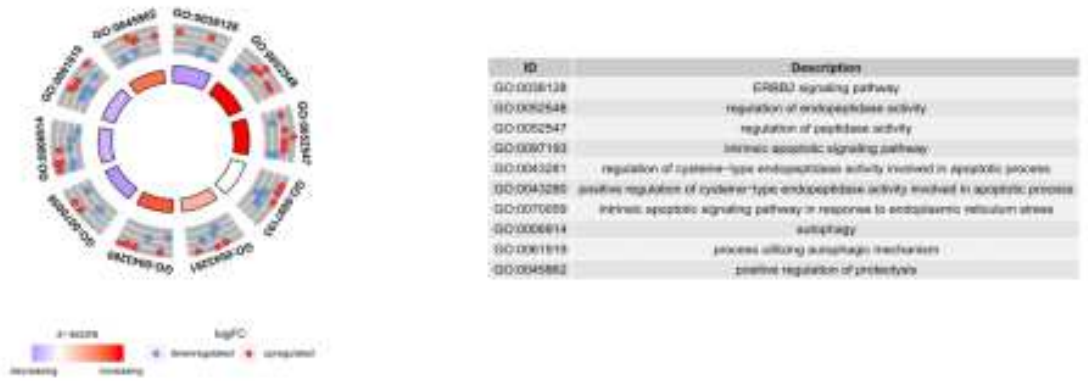

B
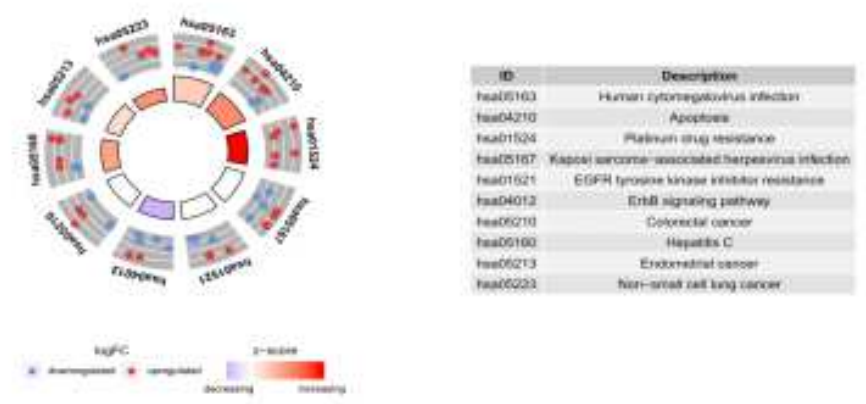

C

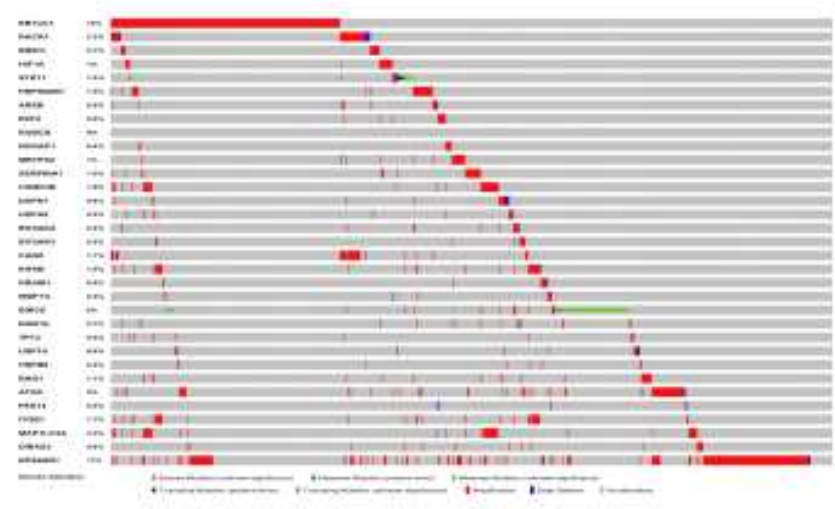

D

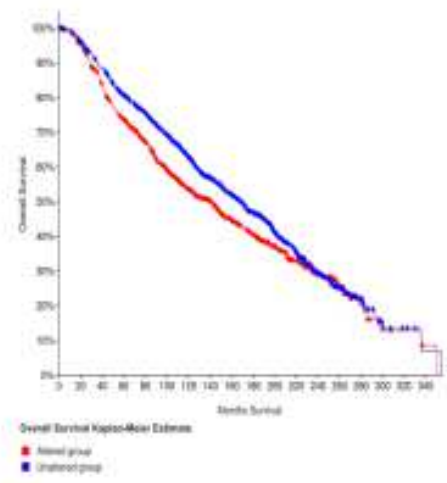

$E$
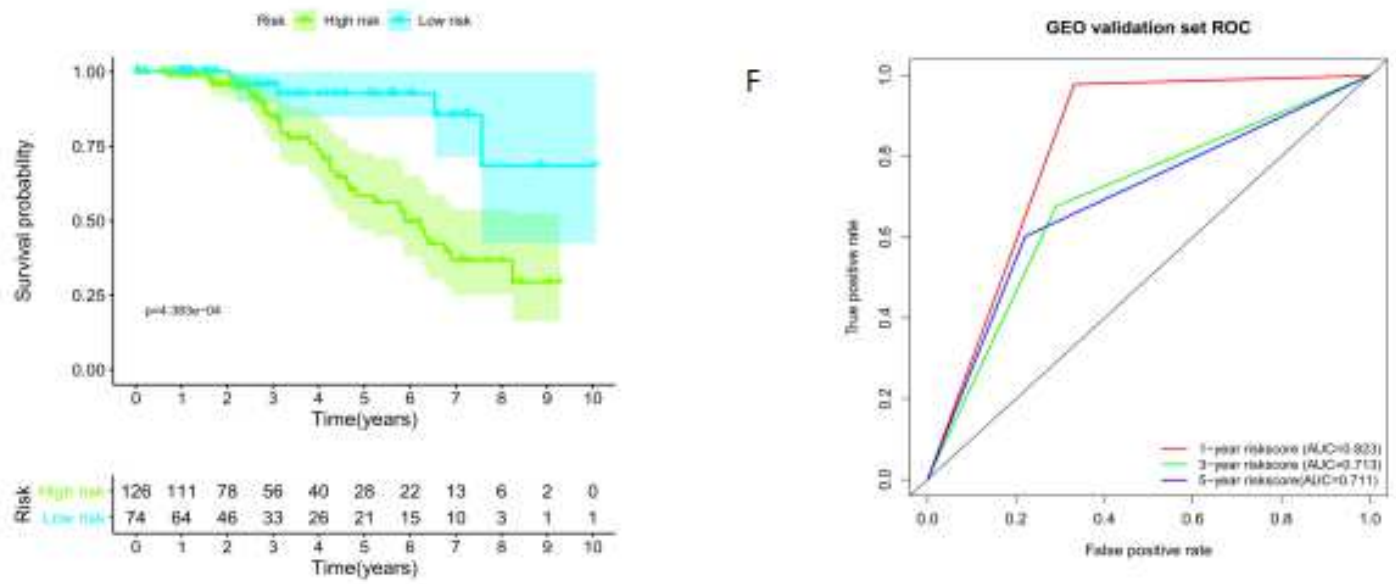

Figure 3 
The genes function enrichment analysis and external validation. (A) GO cricle of genes function. (B) KEGG circle of pathway enrichment analysis. (C) Oncoprint reflecting autophagy genes alteration. (D) Sruvival differentiation among altered group and unaltered group.(E) Survial differentiation between high and low riskscore patients in GEO set.(F) ROC accessment in GEO independent validation cohort.

\section{Supplementary Files}

This is a list of supplementary files associated with this preprint. Click to download.

- table1.doc

- Supplementaryfile.pdf 